\title{
Effect of Common Medications on the Expression of SARS-CoV-2 Entry Receptors in Kidney Tissue
}

\author{
Narjes Saheb Sharif-Askari ${ }^{1}$, Fatemeh Saheb Sharif-Askari ${ }^{1}$, Mashael Alabed ${ }^{1}$, Ahmad Abou Tayoun ${ }^{2,3}$, Tom Loney ${ }^{3}$, \\ Mohammed Uddin ${ }^{3}$, Abiola Senok ${ }^{3}$, Saba Al Heialy ${ }^{3,4}$, Rifat Hamoudi ${ }^{1,5}$, Tarek Kashour ${ }^{6}$, Alawi Alsheikh-Ali ${ }^{3}$, Qutayba Hamid ${ }^{1,4,5}$ \\ and Rabih Halwani ${ }^{1,5,7, *}$
}

Besides the respiratory system, severe acute respiratory syndrome-coronavirus 2 (SARS-CoV-2) infection was shown to affect other essential organs such as the kidneys. Early kidney involvement during the course of infection was associated with worse outcomes, which could be attributed to the direct SARS-CoV-2 infection of kidney cells. In this study, the effect of commonly used medications on the expression of SARS-CoV-2 receptor, angiotensin-converting enzyme (ACE)2, and TMPRSS2 protein in kidney tissues was evaluated. This was done by in silico analyses of publicly available transcriptomic databases of kidney tissues of rats treated with multiple doses of commonly used medications. Of 59 tested medications, $\mathbf{5 6} \%$ modified ACE2 expression, whereas $24 \%$ modified TMPRSS2 expression. ACE2 was increased with only a few of the tested medication groups, namely the renin-angiotensin inhibitors, such as enalapril, antibacterial agents, such as nitrofurantoin, and the proton pump inhibitor, omeprazole. The majority of the other medications decreased ACE2 expression to variable degrees with allopurinol and cisplatin causing the most noticeable downregulation. The expression level of TMPRSS2 was increased with a number of medications, such as diclofenac, furosemide, and dexamethasone, whereas other medications, such as allopurinol, suppressed the expression of this gene. The prolonged exposure to combinations of these medications could regulate the expression of ACE2 and TMPRSS2 in a way that may affect kidney susceptibility to SARS-CoV-2 infection. Data presented here suggest that we should be vigilant about the potential effects of commonly used medications on kidney tissue expression of ACE2 and TMPRSS2.

\section{Study Highlights}

WHAT IS THE CURRENT KNOWLEDGE ON THE TOPIC?

$\square$ Angiotensin-converting enzyme (ACE)2 is the main severe acute respiratory syndrome-coronavirus 2 (SARSCoV-2) entry receptor. SARS-CoV-2 can directly infect and replicate in human kidney cells. Treatment of kidney organoid with human recombinant soluble ACE2 significantly reduced SARS-CoV-2 infection in a dose-dependent manner. It is not known how commonly used medications affect the expression of these receptors in kidney tissue. WHAT QUESTION DID THIS STUDY ADDRESS?

$\checkmark$ Whether commonly used medications regulate expression of SARS-CoV-2 receptors in kidney tissue.

WHAT DOES THIS STUDY ADD TO OUR KNOWLEDGE?

$\square$ Through in silico analyses, we have shown that commonly used medications affect the expression of
SARS-CoV-2 entry receptors in kidney tissue. This may highlight how these medications would affect susceptibility of the kidney to coronavirus disease 2019 infection. HOW MIGHT THIS CHANGE CLINICAL PHARMACOLOGY OR TRANSLATIONAL SCIENCE?

$\checkmark$ Our data suggested that many of the tested medications, or their combinations, may regulate SARS-CoV-2 receptors expression in kidney tissue and may hence modulate its susceptibility to infection. This may suggest that the effect of the medication on SARS-CoV-2 receptors expression may be considered when selecting drug combinations especially for chronic conditions.
Besides the respiratory system, other organs have been shown to be affected by severe acute respiratory syndrome-coronavirus 2 (SARS-CoV-2) infection, such as the gastrointestinal tract, ${ }^{1}$ the liver, ${ }^{2}$ and the kidneys. ${ }^{3}$ Renal impairment has been observed by several studies as a major secondary outcome of coronavirus disease 2019

\footnotetext{
${ }^{1}$ Sharjah Institute of Medical Research, College of Medicine, University of Sharjah, Sharjah, United Arab Emirates; ${ }^{2}$ Al Jalila Genomics Center, Al Jalila Children's Hospital, Dubai, United Arab Emirates; ${ }^{3}$ College of Medicine, Mohammed Bin Rashid University of Medicine and Health Sciences, Dubai, United Arab Emirates; ${ }^{4}$ Meakins-Christie Laboratories, Research Institute of the McGill University Healthy Center, McGill University, Montreal, Quebec, Canada; ${ }^{5}$ Department of Clinical Sciences, College of Medicine, University of Sharjah, Sharjah, United Arab Emirates; ${ }^{6}$ Department of Cardiology, King Fahad Cardiac Center, King Saud University Medical City, Riyadh, Saudi Arabia; ${ }^{7}$ Prince Abdullah Ben Khaled Celiac Disease Research Chair, Department of Pediatrics, Faculty of Medicine, King Saud University, Riyadh, Saudi Arabia. *Correspondence: Rabih Halwani (rhalwani@sharjah.ac.ae)
}

Received: May 8, 2020; accepted: July 17, 2020. doi:10.1111/cts.12862 
(COVID-19) infection, following respiratory dysfunction. ${ }^{4}$ Acute kidney injury during COVID-19 infection could be due to several factors, including the direct infection of renal cells with SARS-CoV-2, cytokine storm, inflammation, and drug-induced toxicity. The progression of renal injury needs to be correlated clinically with the course of COVID-19 to further understand its underlying mechanisms. In a recent observation by Cheng and colleagues, ${ }^{3}$ renal impairment on admission correlated with poor outcomes among patients with COVID-19. The level of renal impairment and elevated baseline serum creatinine were associated with a more severe course of SARS-CoV-2 infection, and with higher likelihood of intensive care unit admission, and mechanical ventilation. This reported increase in serum creatinine early during the infection suggests that it could be attributed to early infection of the kidneys by SARS-CoV-2. On the other hand, late impairments could be a manifestation of systemic inflammation and multi-organ failure or secondary to late infection of the kidneys.

Both SARS-CoV $-1^{5}$ and SARS-CoV- $2^{2}$ were detected in urine samples. Angiotensin-converting enzyme 2 (ACE2) is the main receptor of SARS-CoV-1 and 2, whereas the transmembrane serine protease, TMPRSS2, is needed to prime the viral spike protein, an essential step for virus binding to ACE2 ${ }^{6}$ but the binding affinity of SARS-CoV-2 spike protein to ACE2 is much higher than that of SARS-CoV-1. ${ }^{7}$ CD147 has also been described as an alternative receptor of SARSCoV-2 entry to the cell. ${ }^{8}$

ACE2 was previously shown to be strongly expressed in kidney tubules. ${ }^{9}$ Recently, Monteil and colleagues found that SARS-CoV-2 can directly infect human tubular kidney cells and has the ability to replicate in the kidney organoid postinfection. ${ }^{10}$ They further demonstrated that treatment of kidney organoid with human recombinant soluble ACE2 significantly reduced SARS-CoV-2 infection in a dose-dependent manner. ${ }^{10}$ By binding to SARS-COV-2 spike protein, the recombinant ACE2 is expected to neutralize the viral particles and prevent them from binding to cell surface receptors, and hence lowers infectivity and viral load. That said, the levels of ACE2 expression in the kidney tissues could influence renal susceptibility to SARS-CoV-2 infection. In this study, we set to examine the effect of commonly used medications on the expression of ACE2, TMPRSS2, and CD147 in kidney tissues. By applying bioinformatic analyses of publicly available data, we have determined the ability of 59 commonly used medications to regulate the expression of SARS-CoV-2 receptors in kidney tissues.

\section{METHODS}

Bioinformatic analyses were conducted to evaluate the effect of different groups of medications on mRNA expression levels of ACE2, TMPRSS2, and CD147 gene signatures in rat kidney tissues. Publicly available gene expression datasets on Open Toxicogenomic Project-Genomics Assisted Toxicity Evaluation System ${ }^{11}$ and DrugMatrix toxicogenomic database were used. In these two toxicogenomic projects, rats were treated with different medication groups in biological triplicates for different time points. For the purpose of this study, we selected the longest exposure time available for each medication. From the Toxicogenomic ProjectGenomics Assisted Toxicity Evaluation System study, we used the data of in vivo daily treatments with moderate and high doses for a duration of 29 days. From the DrugMatrix database, we had chosen the daily in vivo treatments that were mainly administered in a single dose manner with the longest post-treatment time points ranging from 1 day to 7 days. Hybridization to the whole genome was performed for all included samples using the RG230_2.0 rat GeneChip (Affymetrix, CA).

Before data preprocessing, all mRNA expression data were evaluated for quality control, and all poor-quality data were removed. The raw Affymetrix data were normalized and log transformed. Microarray data (CEL files) were preprocessed with the Robust Multi-Array Average technique using $\mathrm{R}$ software. ${ }^{12}$ Log-transformed normalized intensities were used in the final analyses; and differentially expressed genes between treated and control samples were carried out using Linear Models for MicroArray data (LIMMA) analyses. ${ }^{13,14}$ Statistical analysis was performed using $R$ (version 3.0.2) and Prism (version 8; GraphPad Software) softwares. For all analyses, $P$ values $<0.05$ were considered significant.

\section{RESULTS AND DISCUSSION}

In this study, bioinformatic analyses of publicly available drug databases were conducted to evaluate the effect of commonly used medications on kidney tissue expression of SARS-CoV-2 receptors (Figure 1). Kidney tissue is known to express ACE2 at higher levels and TMPRSS2 and CD147 at comparable levels to bronchial lung tissue (Figure 1a). Among the viral entry genes, renal expression of ACE2 was affected with a larger number of medications and to a greater extent. Of 59 tested medications, $56 \%$ affected ACE2 (15\% increased and 41\% decreased expression), 27\% affected CD147 (19\% increased and 8\% decreased expression), whereas $24 \%$ affected TMPRSS2 (16\% increased and $8 \%$ decreased expression).

Besides acting as the main receptor for SARS-CoV-1 and SARS-CoV-2, ACE2 enzyme plays an essential role in protecting multiple organs against injury, including the lungs, ${ }^{15}$ the heart, ${ }^{16}$ and the kidneys. ${ }^{17}$ Therefore, although upregulation of ACE2 may increase susceptibility to COVID-19 infection in the early phase of the disease, its suppression could lead to an extensive acute kidney injury at later stages. ${ }^{17}$ In our study, ACE2 was increased with some of the tested medication groups, namely the renin-angiotensin inhibitors, such as enalapril, antibacterial agents, such as nitrofurantoin, and proton pump inhibitor medication, including omeprazole. The majority of the other medications decreased ACE2 expression to variable degrees. The most pronounced downregulation was observed with allopurinol (gout medication) and cisplatin (antineoplastic agent; Figure 1b,c). The average ACE2 fold change caused by each medication class was calculated and displayed in Figure 1b. Interestingly, among the tested analgesics, acetaminophen did not change the ACE2 expression level; whereas multiple nonsteroidal anti-inflammatory drug medications, such as indomethacin and ibuprofen, significantly lowered the mRNA levels of this gene. 
The expression level of TMPRSS2 was increased with a number of medications, such as diclofenac, furosemide, and dexamethasone (Figure 1d). Other medications, such as allopurinol, cisplatin, and acetaminophen, downregulated this membrane-associated enzyme. To our knowledge, this effect of commonly used medications on the renal expression of TMPRSS2 was not reported before. The observed increase in expression of TMPRSS2 will lead to an increase in priming of the virus spike protein and, hence, may enhance the level of SARS-CoV-2 infectivity within this tissue. ${ }^{18}$

We have also tested the effect of these medications on CD147 (Basigin). ${ }^{19}$ Furosemide, piroxicam, prednisolone, and norfloxacin were among the top upregulating medications for CD147 expression, whereas cisplatin, sulindac, and nimesulide were among the top suppressors of its expression (Figure 1e). The regulatory effect of all the different medications on the expression of SARS-CoV-2 receptors is presented in Table 1.

Treatment of common comorbidities, such as hypertension, often requires the chronic use of multiple medications; which may result in an additive regulatory effect on the expression of ACE2 and TMPRSS2.

Given the fact that SARS-CoV-2 infection does not increase the expression of ACE2 and TMPRSS2, ${ }^{6,20,21}$ the combined effect of these chronically used medications may modulate the susceptibility of kidney tissue to SARS-CoV-2 infection. For example, captopril and omeprazole, if used in combination, both increase ACE2 levels. Furosemide increases TMPRSS2 expression, despite lowering the ACE2 level, which may enhance viral priming. On the other hand, a single or combined treatment with penciclovir and acetaminophen, both of which lowers expression of entry receptors, is expected to decrease the availability of receptors for SARS-CoV-2 entry to the cells. Nonetheless, ACE2 plays a protective role against lung and kidney injury ${ }^{22,23}$ and, therefore, the net biological effects of the single or combination drug therapy on the expression of these receptors is still to be elucidated.

Data presented here provides evidence that medications may regulate the expression of SARS-CoV-2 receptors in the kidney tissues and hence may modulate the susceptibility of the kidneys to SASR-CoV-2 infection. The mechanisms regulating drug-induced receptor expression are unknown and requires further investigations. Previous in vitro studies of gene expression changes in response to drugs like cisplatin and ochratoxin $A$ in rat and human kidney cell lines and rat kidney tissue slices mirror the observed in vivo gene expression changes in rats. ${ }^{24-26}$ However, this does not provide proof that those in vivo drug-induced expression changes of SARS-CoV-2 receptors we observed in this bioinformatics-based study would reflect similar changes in humans. Moreover, our results are based on public gene expression (a)

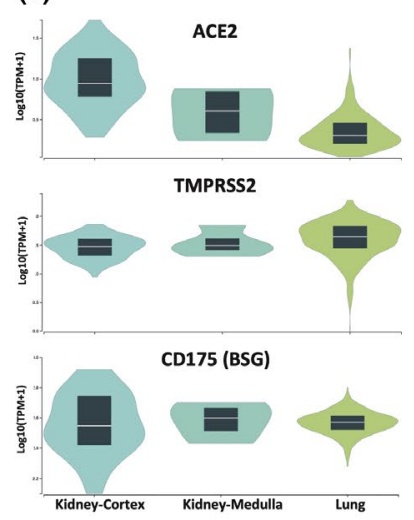

(b)

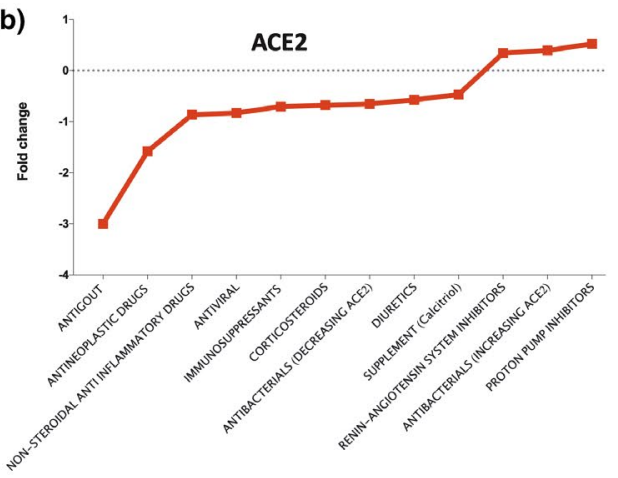

TG-GATE Project Drug Matrix Dataset

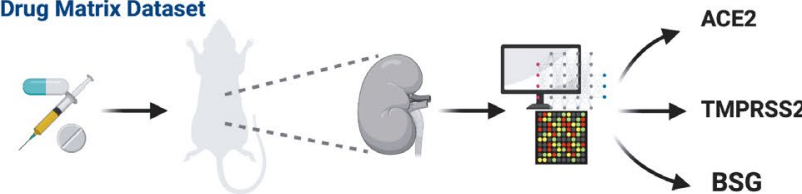

(c)

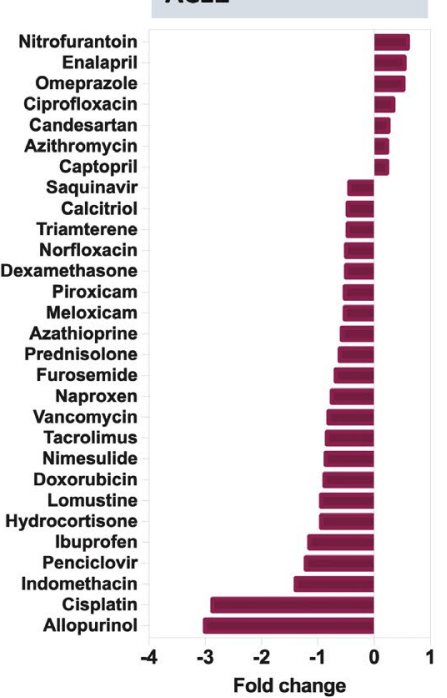

(d)

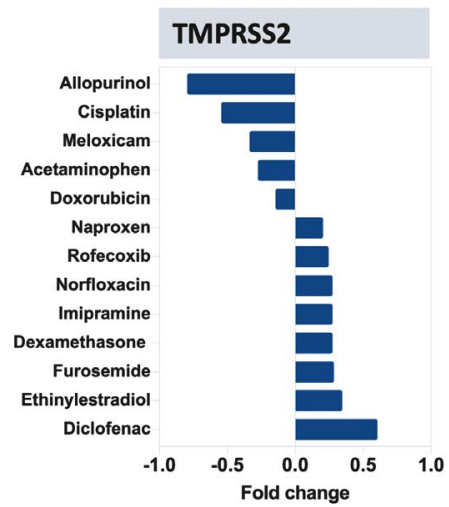

(e)

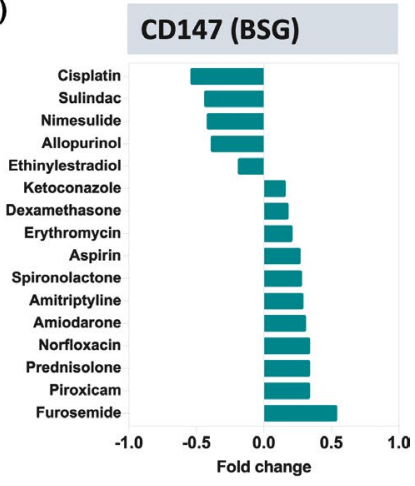

Figure 1 Effect of common medications on the expression levels of severe acute respiratory syndrome-coronavirus 2 entry receptors in kidney. (a) Shows the baseline expression of angiotensin-converting enzyme (ACE)2 and TMPRSS2 in healthy lung and kidney tissues extracted from the Genotype-Tissue Expression project. (b) Represents the main medication classes that affected the expression levels of ACE2. (c-e) Shows medications causing the highest and lowest fold change for ACE2 TMPRSS2, and CD147 expression levels. 
Table 1 Effect of common medications treatments on expression levels of renal SARS-Cov-2 receptors of ACE2 and TMPRSS2

\begin{tabular}{|c|c|c|c|c|c|}
\hline Medication & Classifications & $\begin{array}{c}\text { Repeat dose } \\
\mathrm{mg} / \mathrm{kg}\end{array}$ & $\begin{array}{l}\text { ACE2 mRNA fold } \\
\text { change ( } P \text { value) }\end{array}$ & $\begin{array}{c}\text { TMPRSS2 mRNA } \\
\text { fold change ( } P \\
\text { value) }\end{array}$ & $\begin{array}{c}\text { BSG (CD147) } \\
\text { mRNA fold change } \\
\text { ( } P \text { value) }\end{array}$ \\
\hline \multirow{2}{*}{$\begin{array}{l}\text { Acetaminophen, } \\
29 \text { days, oral }\end{array}$} & Analgesics & 600 & Nonsignificant & $-0.26(P=0.002)^{\star \star}$ & Nonsignificant \\
\hline & Analgesics & 1,000 & Nonsignificant & Nonsignificant & Nonsignificant \\
\hline \multirow[t]{3}{*}{ Captopril, 29 days, oral } & $\begin{array}{l}\text { Renin-angiotensin } \\
\text { system inhibitors }\end{array}$ & 100 & Nonsignificant & Nonsignificant & Nonsignificant \\
\hline & $\begin{array}{l}\text { Renin-angiotensin } \\
\text { system inhibitors }\end{array}$ & 300 & $0.23(P=0.045)^{\star}$ & Nonsignificant & Nonsignificant \\
\hline & $\begin{array}{l}\text { Renin-angiotensin } \\
\text { system inhibitors }\end{array}$ & 1,000 & Nonsignificant & Nonsignificant & Nonsignificant \\
\hline Captopril, 5 days, oral & $\begin{array}{l}\text { Renin-angiotensin } \\
\text { system inhibitors }\end{array}$ & 1,750 & Nonsignificant & Nonsignificant & Nonsignificant \\
\hline $\begin{array}{l}\text { Candesartan, } 3 \text { days, } \\
\text { oral }\end{array}$ & $\begin{array}{l}\text { Renin-angiotensin } \\
\text { system inhibitors }\end{array}$ & 1,300 & $0.25(P=0.021)^{\star}$ & Nonsignificant & Nonsignificant \\
\hline \multirow[t]{2}{*}{ Enalapril, 29 days, oral } & $\begin{array}{l}\text { Renin-angiotensin } \\
\text { system inhibitors }\end{array}$ & 300 & Nonsignificant & Nonsignificant & Nonsignificant \\
\hline & $\begin{array}{l}\text { Renin-angiotensin } \\
\text { system inhibitors }\end{array}$ & 600 & $0.54(P<0.0001)^{\star \star \star \star}$ & Nonsignificant & Nonsignificant \\
\hline Ramipril, 3 days, oral & $\begin{array}{l}\text { Renin-angiotensin } \\
\text { system inhibitors }\end{array}$ & 1,500 & Nonsignificant & Nonsignificant & Nonsignificant \\
\hline Spironolactone, 5 days & Diuretics & 300 & Nonsignificant & Nonsignificant & $0.27(P=0.002)^{\star \star}$ \\
\hline \multirow{2}{*}{$\begin{array}{l}\text { Triamterene, } 29 \text { days, } \\
\text { oral }\end{array}$} & Diuretics & 50 & Nonsignificant & Nonsignificant & Nonsignificant \\
\hline & Diuretics & 150 & $-0.47(P=0.002)^{\star \star}$ & Nonsignificant & Nonsignificant \\
\hline $\begin{array}{l}\text { Furosemide, } 5 \text { days, } \\
\text { oral }\end{array}$ & Diuretics & 375 & $-0.68(P=0.006)^{\star \star}$ & $0.27(P=0.01)^{\star}$ & $0.53(P=0.0001)^{\star \star \star}$ \\
\hline Diclofenac, 5 days, oral & $\begin{array}{l}\text { Analgesics , nonsteroidal } \\
\text { anti-inflammatory drugs }\end{array}$ & 3.5 & Nonsignificant & $0.59(P=0.0003)^{\star \star \star}$ & Nonsignificant \\
\hline Ibuprofen, 5 days, oral & $\begin{array}{l}\text { Analgesics , nonsteroidal } \\
\text { anti-inflammatory drugs }\end{array}$ & 263 & $-1.15(P=0.048)^{\star \star}$ & Nonsignificant & Nonsignificant \\
\hline \multirow[t]{2}{*}{$\begin{array}{l}\text { Indomethacin, } 29 \text { days, } \\
\text { oral }\end{array}$} & $\begin{array}{l}\text { Analgesics , nonsteroidal } \\
\text { anti-inflammatory drugs }\end{array}$ & 1.6 & Nonsignificant & Nonsignificant & Nonsignificant \\
\hline & $\begin{array}{l}\text { Analgesics > nonsteroidal } \\
\text { anti-inflammatory drugs }\end{array}$ & 5 & $-1.39(P=0.048)^{\star \star}$ & Nonsignificant & Nonsignificant \\
\hline $\begin{array}{l}\text { Mefenamic acid, } \\
5 \text { days, oral }\end{array}$ & $\begin{array}{l}\text { Analgesics , nonsteroidal } \\
\text { anti-inflammatory drugs }\end{array}$ & 93 & Nonsignificant & Nonsignificant & Nonsignificant \\
\hline Meloxicam, 1 day, oral & $\begin{array}{l}\text { Analgesics , nonsteroidal } \\
\text { anti-inflammatory drugs }\end{array}$ & 33 & $-0.52(P=0.0009)^{\star \star \star}$ & $-0.32(P=0.006)^{\star \star}$ & Nonsignificant \\
\hline Piroxicam, 5 days, oral & $\begin{array}{l}\text { Analgesics , nonsteroidal } \\
\text { anti-inflammatory drugs }\end{array}$ & 14 & $-0.52(P=0.01)^{\star}$ & Nonsignificant & $0.33(P=0.0008)$ \\
\hline Naproxen, 1 day, oral & $\begin{array}{l}\text { Analgesics , nonsteroidal } \\
\text { anti-inflammatory drugs }\end{array}$ & 134 & $-0.75(P=0.002)^{\star \star}$ & $0.19(P=0.022)^{\star}$ & Nonsignificant \\
\hline $\begin{array}{l}\text { Nimesulide, } 5 \text { days, } \\
\text { oral }\end{array}$ & $\begin{array}{l}\text { Analgesics , nonsteroidal } \\
\text { anti-inflammatory drugs }\end{array}$ & 162 & $-0.86(P=0.02)^{\star \star}$ & Nonsignificant & $-0.41(P=0.001)^{\star \star}$ \\
\hline \multirow[t]{2}{*}{ Sulindac, 1 day, oral } & $\begin{array}{l}\text { Analgesics , nonsteroidal } \\
\text { anti-inflammatory drugs }\end{array}$ & 23 & Nonsignificant & Nonsignificant & Nonsignificant \\
\hline & $\begin{array}{l}\text { Analgesics > nonsteroidal } \\
\text { anti-inflammatory drugs }\end{array}$ & 64 & Nonsignificant & Nonsignificant & $-0.43(P=0.002)^{\star \star}$ \\
\hline Rofecoxib, 1 day, oral & $\begin{array}{l}\text { Selective nonsteroidal } \\
\text { anti-inflammatory drugs }\end{array}$ & 755 & Nonsignificant & $0.23(P=0.006)^{\star \star}$ & Nonsignificant \\
\hline $\begin{array}{l}\text { Dexamethasone, } \\
3 \text { days, oral }\end{array}$ & Corticosteroids & 300 & $-0.50(P=0.022)^{\star}$ & $0.26(P=0.013)^{\star}$ & $0.17(P=0.032)^{\star}$ \\
\hline $\begin{array}{l}\text { Hydrocortisone, } \\
5 \text { days, s.c. }\end{array}$ & Corticosteroids & 65 & $-0.94(P=0.002)^{\star \star}$ & Nonsignificant & Nonsignificant \\
\hline $\begin{array}{l}\text { Prednisolone, } 1 \text { day, } \\
\text { oral }\end{array}$ & Corticosteroids & 184 & $-0.61(P<0.0001)^{\star \star \star \star}$ & Nonsignificant & $0.33(P=0.005)^{\star \star}$ \\
\hline \multirow{2}{*}{$\begin{array}{l}\text { Methyltestosterone, } \\
29 \text { days, oral }\end{array}$} & Androgens & 100 & $0.42(P=0.0007)^{\star \star \star}$ & Nonsignificant & Nonsignificant \\
\hline & Androgens & 300 & $0.37(P=0.006)^{\star \star}$ & Nonsignificant & Nonsignificant \\
\hline \multirow{2}{*}{$\begin{array}{l}\text { Ethinylestradiol, } \\
29 \text { days, oral }\end{array}$} & Contraceptives & 3 & Nonsignificant & $0.33(P=0.0004)^{\star \star *}$ & $-0.18(P=0.03)^{\star}$ \\
\hline & Contraceptives & 10 & Nonsignificant & $0.32(P=0.002)^{\star \star}$ & Nonsignificant \\
\hline
\end{tabular}




\begin{tabular}{|c|c|c|c|c|c|}
\hline Medication & Classifications & $\begin{array}{l}\text { Repeat dose } \\
\mathrm{mg} / \mathrm{kg}\end{array}$ & $\begin{array}{l}\text { ACE2 mRNA fold } \\
\text { change ( } P \text { value) }\end{array}$ & $\begin{array}{c}\text { TMPRSS2 mRNA } \\
\text { fold change ( } P \\
\text { value) }\end{array}$ & $\begin{array}{c}\text { BSG (CD147) } \\
\text { mRNA fold change } \\
\text { ( } P \text { value })\end{array}$ \\
\hline \multirow{2}{*}{$\begin{array}{l}\text { Omeprazole, } 29 \text { days, } \\
\text { oral }\end{array}$} & Proton pump inhibitors & 300 & $0.52(P=0.001)^{\star \star}$ & Nonsignificant & Nonsignificant \\
\hline & Proton pump inhibitors & 1,000 & Nonsignificant & Nonsignificant & Nonsignificant \\
\hline Penciclovir, 5 days, s.c. & Antiviral & 1,200 & $-1.21(P=0.013)^{\star}$ & Nonsignificant & Nonsignificant \\
\hline Saquinavir_1d_Oral & Antiviral & 1,200 & $-0.44(P=0.0002)^{\star}$ & Nonsignificant & Nonsignificant \\
\hline Ritonavir, $1 \mathrm{~d}$, Oral & Antiviral & 1,200 & Nonsignificant & Nonsignificant & Nonsignificant \\
\hline Gentamicin, 29d, oral & Antibacterials & 30,100 & Nonsignificant & Nonsignificant & Nonsignificant \\
\hline $\begin{array}{l}\text { Vancomycin, } 5 \text { days, } \\
\text { i.v. }\end{array}$ & Antibacterials & 160 & $-0.81(P=0.002)^{\star \star}$ & Nonsignificant & Nonsignificant \\
\hline Amikacin, 5 days, i.p. & Antibacterials & 160 & Nonsignificant & Nonsignificant & Nonsignificant \\
\hline \multirow{2}{*}{$\begin{array}{l}\text { Ciprofloxacin, } 29 \text { days, } \\
\text { oral }\end{array}$} & Antibacterials & 300 & $0.34(P=0.008)^{\star \star}$ & Nonsignificant & Nonsignificant \\
\hline & Antibacterials & 1,000 & $0.24(P=0.04)^{\star}$ & Nonsignificant & Nonsignificant \\
\hline $\begin{array}{l}\text { Norfloxacin, } 5 \text { days, } \\
\text { oral }\end{array}$ & Antibacterials & 1,500 & $-0.5(P=0.004)^{\star \star}$ & $0.26(P=0.007)^{\star \star}$ & $0.33(P=0.0007)^{\star \star \star}$ \\
\hline $\begin{array}{l}\text { Cephalexin, } 3 \text { days, } \\
\text { oral }\end{array}$ & Antibacterials & 2,500 & Nonsignificant & Nonsignificant & Nonsignificant \\
\hline Azithromycin, 5 days & Antibacterials & 225 & $0.23(P=0.04)^{\star}$ & Nonsignificant & Nonsignificant \\
\hline \multirow{2}{*}{$\begin{array}{l}\text { Erythromycin } \\
\text { ethylsuccinate, } \\
29 \text { days, oral }\end{array}$} & Antibacterials & 300 & Nonsignificant & Nonsignificant & $0.11(P=0.024)^{\star}$ \\
\hline & Antibacterials & 1,000 & Nonsignificant & Nonsignificant & $0.2(P=0.003)^{\star *}$ \\
\hline \multirow{2}{*}{$\begin{array}{l}\text { Nitrofurantoin, } 29 \text { days, } \\
\text { oral }\end{array}$} & Antibacterials & 30 & Nonsignificant & Nonsignificant & Nonsignificant \\
\hline & Antibacterials & 100 & $0.6(P=0.02)^{\star}$ & Nonsignificant & Nonsignificant \\
\hline \multirow{2}{*}{$\begin{array}{l}\text { Ketoconazole, } 29 \text { days, } \\
\text { oral }\end{array}$} & Antifungals & 30 & Nonsignificant & Nonsignificant & Nonsignificant \\
\hline & Antifungals & 100 & Nonsignificant & Nonsignificant & $0.15(P=0.04)^{\star}$ \\
\hline $\begin{array}{l}\text { Ethambutol, } 3 \text { days, } \\
\text { oral }\end{array}$ & Antimycobacterials & 998 & Nonsignificant & Nonsignificant & Nonsignificant \\
\hline $\begin{array}{l}\text { Rifampicin, } 29 \text { days, } \\
\text { oral }\end{array}$ & Antimycobacterials & 60,200 & Nonsignificant & Nonsignificant & Nonsignificant \\
\hline $\begin{array}{l}\text { Cyclophosphamide, } \\
29 \text { days, oral }\end{array}$ & Antineoplastic drugs & 5,15 & Nonsignificant & Nonsignificant & Nonsignificant \\
\hline \multirow[t]{3}{*}{ Cisplatin, 29 days, oral } & Antineoplastic drugs & 0.1 & Nonsignificant & Nonsignificant & Nonsignificant \\
\hline & Antineoplastic drugs & 0.3 & $-0.83(P=0.0005)^{\star \star \star}$ & Nonsignificant & Nonsignificant \\
\hline & Antineoplastic drugs & 1 & $-2.87(P<0.0001)^{\star \star \star \star}$ & $\begin{array}{c}-0.53 \\
(P<0.0001)^{\star \star \star \star}\end{array}$ & $\begin{array}{c}-0.53 \\
(P<0.0001)^{\star \star \star \star}\end{array}$ \\
\hline \multirow{2}{*}{$\begin{array}{l}\text { Lomustine, } 29 \text { days, } \\
\text { oral }\end{array}$} & Antineoplastic drugs & 2 & Nonsignificant & Nonsignificant & Nonsignificant \\
\hline & Antineoplastic drugs & 6 & $-0.94(P<0.0001)^{\star \star \star \star}$ & Nonsignificant & Nonsignificant \\
\hline \multirow{2}{*}{$\begin{array}{l}\text { Doxorubicin, } 29 \text { days, } \\
\text { oral }\end{array}$} & Antineoplastic drugs & 0.3 & Nonsignificant & $-0.13(P=0.038)^{*}$ & Nonsignificant \\
\hline & Antineoplastic drugs & 1 & $-0.88(P=0.026)^{\star \star \star}$ & Nonsignificant & Nonsignificant \\
\hline \multirow{2}{*}{$\begin{array}{l}\text { Imipramine, } 29 \text { days, } \\
\text { oral }\end{array}$} & Antidepressants & 30 & $-0.30(P=0.002)^{\star \star}$ & Nonsignificant & Nonsignificant \\
\hline & Antidepressants & 100 & Nonsignificant & $0.26(P=0.009)^{\star \star}$ & Nonsignificant \\
\hline $\begin{array}{l}\text { Amitriptyline, } 1 \text { day, } \\
\text { oral }\end{array}$ & Antidepressants & 160 & Nonsignificant & Nonsignificant & $0.28(P=0.04)^{\star \star}$ \\
\hline \multirow{2}{*}{$\begin{array}{l}\text { Valproic acid, } 29 \text { days, } \\
\text { oral }\end{array}$} & Antiepileptics & 150 & Nonsignificant & Nonsignificant & Nonsignificant \\
\hline & Antiepileptics & 450 & $-0.3(P=0.042)^{\star \star}$ & Nonsignificant & Nonsignificant \\
\hline \multirow{2}{*}{$\begin{array}{l}\text { Allopurinol, } 29 \text { days, } \\
\text { oral }\end{array}$} & Antigout & 50 & Nonsignificant & Nonsignificant & Nonsignificant \\
\hline & Antigout & 150 & $-3(P<0.0001)^{\star \star \star \star}$ & $-0.78(P=0.004)^{\star \star}$ & $-0.38(P=0.018)^{\star}$ \\
\hline $\begin{array}{l}\text { Azathioprine, } 5 \text { days, } \\
\text { oral }\end{array}$ & Immunosuppressants & 54 & $-0.57(P=0.003)^{\star \star}$ & Nonsignificant & Nonsignificant \\
\hline $\begin{array}{l}\text { Methotrexate, } 3 \text { days, } \\
\text { oral }\end{array}$ & Immunosuppressants & 27 & Nonsignificant & Nonsignificant & Nonsignificant \\
\hline $\begin{array}{l}\text { Tacrolimus, } 5 \text { days, } \\
\text { oral }\end{array}$ & Immunosuppressants & 134 & $-0.84(P<0.0001)^{\star \star \star \star}$ & Nonsignificant & Nonsignificant \\
\hline $\begin{array}{l}\text { Cyclosporin, } 29 \text { days, } \\
\text { oral }\end{array}$ & Immunosuppressants & 30,100 & Nonsignificant & Nonsignificant & Nonsignificant \\
\hline Aspirin, 1 day, oral & Antiplatelet drugs & 375 & Nonsignificant & Nonsignificant & $0.26(P=0.01)^{\star}$ \\
\hline
\end{tabular}




\begin{tabular}{|c|c|c|c|c|c|}
\hline Medication & Classifications & $\begin{array}{c}\text { Repeat dose } \\
\mathrm{mg} / \mathrm{kg}\end{array}$ & $\begin{array}{l}\text { ACE2 mRNA fold } \\
\text { change ( } P \text { value) }\end{array}$ & $\begin{array}{c}\text { TMPRSS2 mRNA } \\
\text { fold change ( } P \\
\text { value) }\end{array}$ & $\begin{array}{c}\text { BSG (CD147) } \\
\text { mRNA fold change } \\
\text { ( } P \text { value) }\end{array}$ \\
\hline $\begin{array}{l}\text { Amiodarone, } 5 \text { days, } \\
\text { oral }\end{array}$ & Antiarrhythmics & 147 & Nonsignificant & Nonsignificant & $0.3(P=0.006)^{\star}$ \\
\hline $\begin{array}{l}\text { Fenofibrate, } 5 \text { days, } \\
\text { oral }\end{array}$ & Lipid modifying drugs & 215 & Nonsignificant & Nonsignificant & Nonsignificant \\
\hline $\begin{array}{l}\text { Gemfibrozil, } 7 \text { days, } \\
\text { oral }\end{array}$ & Lipid modifying drugs & 700 & Nonsignificant & Nonsignificant & Nonsignificant \\
\hline $\begin{array}{l}\text { Clofibrate, } 29 \text { days, } \\
\text { oral }\end{array}$ & Lipid modifying drugs & 100,300 & Nonsignificant & Nonsignificant & Nonsignificant \\
\hline $\begin{array}{l}\text { Atorvastatin, } 3 \text { days, } \\
\text { oral }\end{array}$ & Lipid modifying drugs & 300 & Nonsignificant & Nonsignificant & Nonsignificant \\
\hline Calcitriol, 5 days, oral & Supplement & 0.04 & $-0.47(P=0.009)^{\star \star}$ & Nonsignificant & Nonsignificant \\
\hline
\end{tabular}

Effect of medications on expression of ACE2 and TMPRSS2 was measured with two controls vs. two treatments per dose.For all analyses, $p<0.05$ was considered significant, presented as bold value. ${ }^{*} \mathrm{P}<0.05$, ${ }^{\star *} \mathrm{P}<0.01$, ${ }^{\star \star *} \mathrm{P}<0.001,{ }^{\star \star \star *} \mathrm{P}<0.0001$.

ACE2, angiotensin-converting enzyme; BSG, Basigin; SARS-CoV-2, severe acute respiratory syndrome-coronavirus 2.

For all analyses, $P<0.05$ was considered significant, presented as bold value.

${ }^{*} P<0.05$;

${ }^{\star *} P<0.01$;

${ }^{* * *} P<0.001 ;$

${ }^{\star \star \star \star} P<0.0001$

datasets and thus they may or may not reflect changes in protein expression. Therefore, confirmatory experiments at the mRNA and protein levels are needed to support our findings. Additionally, population studies are necessary to ultimately understand the clinical implications of these findings and further guide the proper use of these medications or identify safer alternatives.

\section{Acknowledgments.}

None.

Conflict of Interest. The authors declared no competing interests for this work.

Funding. This research has been financially supported by Tissue Injury and Repair (TIR) group operational grant (Grant code: 150317); COVID-19 research grant; seed grant (Grant code: 2001090275); and by collaborative research grant (Grant code: 2001090278) to RH, University of Sharjah, UAE; and by a Sandooq AI Watan Applied Research \& Development grant to $\mathrm{RH}$; and by Prince Abdullah Ben Khalid Celiac Disease Research Chair, under the Vice Deanship of Research Chairs, King Saud University, Riyadh, Kingdom of Saudi Arabia.

Author Contributions. N.S.A., A.A.T., R.H., A.A., and R.H. wrote the manuscript. R.H., N.S.A., F.S.A., Q.H., A.A.T., T.L., M.U., and T.K. designed the research. N.S.A., F.S.A., M.A., and R.H. performed the research. N.S.A., F.S.A., M.A., A.S., and S.A. analyzed the data.

1. Xiao, F. etal. Evidence for gastrointestinal infection of SARS-CoV-2. Gastroenterology 158, 1831-1833.e1833 (2020)

2. Huang, C. et al. Clinical features of patients infected with 2019 novel coronavirus in Wuhan, China. Lancet 395, 497-506 (2020)

3. Cheng, Y. et al. Kidney disease is associated with in-hospital death of patients with COVID-19. Kidney Int. 97, 829-838 (2020).

4. Ronco, C., Reis, T. \& Husain-Syed, F. Management of acute kidney injury in patients with COVID-19. Lancet Resp Med. 8, 738-742 (2020).

5. Peiris, J.S. et al. Clinical progression and viral load in a community outbreak of coronavirus-associated SARS pneumonia: a prospective study. Lancet 361, 17671772 (2003).
6. Hoffmann, M. et al. SARS-CoV-2 cell entry depends on ACE2 and TMPRSS2 and is blocked by a clinically proven protease inhibitor. Cell 181, 271-280.e278 (2020).

7. Walls, A.C. et al. Structure, function, and antigenicity of the SARS-CoV-2 spike glycoprotein. Cell 181, 281-292.e286 (2020).

8. Ulrich, H. \& Pillat, M.M. CD147 as a target for COVID-19 treatment: suggested effects of azithromycin and stem cell engagement. Stem Cell Rev. Rep. 16, 434-440 (2020).

9. Danilczyk, U. \& Penninger, J.M. Angiotensin-converting enzyme II in the heart and the kidney. Circ. Res. 98, 463-471 (2006)

10. Monteil, V. et al. Inhibition of SARS-CoV-2 infections in engineered human tissues using clinical-grade soluble human ACE2. Cell 181, 905-913.e907 (2020).

11. Igarashi, Y. et al. Open TG-GATES: a large-scale toxicogenomics database. Nucleic Acids Res. 43, D921-D927 (2015).

12. Hughey, J.J. \& Butte, A.J. Robust meta-analysis of gene expression using the elastic net. Nucleic Acids Res. 43, e79 (2015).

13. Dudoit, S., Yang, Y.H., Callow, M.J. \& Speed, T.P. Statistical methods for identifying differentially expressed genes in replicated cDNA microarray experiments. Stat. Sin. 12, 111-139 (2002)

14. Smyth Gordon, K. Linear models and empirical Bayes methods for assessing differential expression in microarray experiments. Stat. Appl. Genet. Mol. Biol. 3, 1-25 (2004).

15. Rey-Parra, G.J. et al. Angiotensin converting enzyme 2 abrogates bleomycin-induced lung injury. J. Mol. Med. 90, 637-647 (2012).

16. Patel, V.B., Zhong, J.-C., Grant, M.B. \& Oudit, G.Y. Role of the ACE2/angiotensin 1-7 axis of the renin-angiotensin system in heart failure. Circ. Res. 118, 1313-1326 (2016).

17. Wong, D.W. et al. Loss of angiotensin-converting enzyme-2 (Ace2) accelerates diabetic kidney injury. Am. J. Pathol. 171, 438-451 (2007).

18. Wambier, C.G. \& Goren, A. Severe acute respiratory syndrome coronavirus 2 (SARS-CoV-2) infection is likely to be androgen mediated. J. Am. Acad. Dermatol. 83, 308-309 (2020)

19. Wang, K. et al. SARS-CoV-2 invades host cells via a novel route: CD147-spike protein. bioRxiv. https://doi.org/10.1101/2020.03.14.988345

20. Burgueño, J.F. et al. Expression of SARS-CoV-2 entry molecules ACE2 and TMPRSS2 in the gut of patients with IBD. Inflamm. Bowel Dis. 26, 797-808 (2020).

21. Saheb Sharif-Askari, N. et al. Airways expression of SARS-CoV-2 receptor, ACE2, and TMPRSS2 is lower in children than adults and increases with smoking and COPD. Mol. Therapy Meth. Clin. Dev. 18, 1-6 (2020).

22. Kuba, K. et al. A crucial role of angiotensin converting enzyme 2 (ACE2) in SARS coronavirus-induced lung injury. Nat. Med. 11, 875-879 (2005).

23. Cheng, H., Wang, Y. \& Wang, G.-Q. Organ-protective effect of angiotensin-converting enzyme 2 and its effect on the prognosis of COVID-19. J. Med. Virol. 92 , 726-730 (2020).

24. Azad, M.A.K. et al. Polymyxin B induces apoptosis in kidney proximal tubular cells. Antimicrob. Agents Chemother. 57, 4329 (2013).

25. Vickers, A.E. et al. Kidney slices of human and rat to characterize cisplatin-induced injury on cellular pathways and morphology. Toxicol. Pathol. 32, 577-590 (2004). 
26. Jennings, P. et al. Transcriptomic alterations induced by ochratoxin A in rat and human renal proximal tubular in vitro models and comparison to a rat in vivo model. Arch. Toxicol. 86, 571-589 (2012).

(C) 2020 The Authors. Clinical and Translational Science published by Wiley Periodicals LLC on behalf of the American Society for Clinical Pharmacology and
Therapeutics. This is an open access article under the terms of the Creative Commons AttributionNonCommercial-NoDerivs License, which permits use and distribution in any medium, provided the original work is properly cited, the use is non-commercial and no modifications or adaptations are made. 